\title{
A!
}

This is an electronic reprint of the original article.

This reprint may differ from the original in pagination and typographic detail.

Ockeloen-Korppi, Caspar; Damskägg, Erno; Paraoanu, Gheorghe-Sorin; Massel, Francesco; Sillanpää, Mika

Revealing hidden quantum correlations in an electromechanical measurement

Published in:

Physical Review Letters

Published: 14/12/2018

Document Version

Publisher's PDF, also known as Version of record

Please cite the original version:

Ockeloen-Korppi, C., Damskägg, E., Paraoanu, G-S., Massel, F., \& Sillanpää, M. (2018). Revealing hidden quantum correlations in an electromechanical measurement. Physical Review Letters, 121(24), [243601]. https://journals.aps.org/prl/abstract/10.1103/PhysRevLett.121.243601

This material is protected by copyright and other intellectual property rights, and duplication or sale of all or part of any of the repository collections is not permitted, except that material may be duplicated by you for your research use or educational purposes in electronic or print form. You must obtain permission for any other use. Electronic or print copies may not be offered, whether for sale or otherwise to anyone who is not an authorised user. 


\title{
Revealing Hidden Quantum Correlations in an Electromechanical Measurement
}

\author{
C. F. Ockeloen-Korppi, ${ }^{1}$ E. Damskägg, ${ }^{1}$ G. S. Paraoanu, ${ }^{1}$ F. Massel, ${ }^{2}$ and M. A. Sillanpää ${ }^{1, *}$ \\ ${ }^{1}$ Department of Applied Physics, Aalto University, P.O. Box 15100, FI-00076 AALTO, Finland \\ ${ }^{2}$ Department of Physics and Nanoscience Center, University of Jyväskylä, P.O. Box 35 (YFL), \\ FI-40014 University of Jyväskylä, Finland
}

(Received 29 August 2018; published 14 December 2018)

\begin{abstract}
Under a strong quantum measurement, the motion of an oscillator is disturbed by the measurement backaction, as required by the Heisenberg uncertainty principle. When a mechanical oscillator is continuously monitored via an electromagnetic cavity, as in a cavity optomechanical measurement, the backaction is manifest by the shot noise of incoming photons that becomes imprinted onto the motion of the oscillator. Following the photons leaving the cavity, the correlations appear as squeezing of quantum noise in the emitted field. Here we observe such "ponderomotive" squeezing in the microwave domain using an electromechanical device made out of a superconducting resonator and a drumhead mechanical oscillator. Under a strong measurement, the emitted field develops complex-valued quantum correlations, which in general are not completely accessible by standard homodyne measurements. We recover these hidden correlations, using a phase-sensitive measurement scheme employing two local oscillators. The utilization of hidden correlations presents a step forward in the detection of weak forces, as it allows us to fully utilize the quantum noise reduction under the conditions of strong force sensitivity.
\end{abstract}

DOI: 10.1103/PhysRevLett.121.243601

Squeezed states of the propagating electromagnetic field form a fundamental group of nonclassical states $[1,2]$. In a squeezed state, fluctuations of the field in a certain quadrature of the oscillations are diminished below the level of vacuum fluctuations. Such a property of low noise has raised long-standing interest in precision measurements in optics [3-7] or more recently in the microwave frequency domain [8-10], since in several detection applications the sensitivity is limited by photon shot noise. This is the situation particularly in the emerging field of gravitational astronomy, where squeezed light will become an indispensable asset in the near future [11,12]. Furthermore, quantum information processing with continuous variables utilizes multimode squeezed states as the essential resource [13-15].

On the other hand, squeezed electromagnetic fields are connected to intriguing physics. A squeezed environment can suppress relaxation [16], or it can reveal new phenomena $[9,17,18]$. Various nonlinear optical processes such as four-wave mixing or parametric oscillations can produce squeezed light [19-23]. Following the early work [24,25], itinerant microwaves are nowadays routinely squeezed using Josephson parametric amplifiers (JPA) at deep cryogenic temperatures [26-31].

Cavity optomechanics, which studies the interaction of electromagnetic fields and mechanical oscillations, provides a novel platform to produce squeezed light. The necessary nonlinear mechanism is provided by the radiation-pressure interaction that couples cavity energy to mechanical displacement. Squeezing in optomechanical cavities $[32,33]$ arises under an intense measurement that couples amplitude fluctuations of an incoming laser to phase fluctuations of the output field. Such "ponderomotive squeezing" has recently been produced in several experiments in optics [34-39]. In the microwave regime, we mention the realization of strong measurements [40-42] and squeezed microwaves obtained via degenerate parametric amplification [43].

In the present work, we show how certain complexvalued quantum correlations, which are hidden from standard homodyne detection, can be recovered by the use of two sinusoidal local oscillators, as recently proposed theoretically by Buchmann et al. [44]. Besides fundamental interest, these hidden correlations are relevant for sensitive measurements since they appear under the condition where the system is the most responsive to forces. Furthermore, as a test bed for this detection setup, we create ponderomotive squeezing in the microwave frequency regime, thereby demonstrating a new approach to create squeezed microwaves, distinct from JPA or from that realized in Ref. [43].

At optical frequencies, squeezing is detected using homodyne detection. Within the theoretical framework introduced by Glauber [45], photodetectors are sensitive to the even normal-ordered correlators of the electromagnetic field. In order to measure the expectation values of a general quadrature operator $X^{\theta}(\omega)=\frac{1}{2}\left(a^{\dagger} e^{i \theta}+a e^{-i \theta}\right)$ and its correlation functions, it is necessary to mix the incoming signal with a local field $b$ [the local oscillator (LO)]. Depending on the state of the local field, it is possible to access the expectation value of the original quadratures 
through an intensity measurement of the mixed signal. The inclusion of low-pass filters in the measurement process allows us to extend the applicability of this description to the microwave regime.

In the standard (balanced) homodyne detection setup, the LO is chosen to be in a coherent state at a specific frequency, i.e., $\langle b\rangle=\alpha_{0} \exp \left(-i \omega_{0} t\right)$. In this case, it is possible to show that homodyne detection allows access to the following frequency-domain correlator

$$
\begin{aligned}
S_{X}^{\theta}(\omega)= & S_{X}(\omega) \cos ^{2} \theta+S_{Y}(\omega) \sin ^{2} \theta \\
& +2 \operatorname{Re}\left[S_{X Y}(\omega)\right] \sin \theta \cos \theta .
\end{aligned}
$$

Here, $S_{X}(\omega)=\frac{1}{2}\langle\{X(\omega), X(-\omega)\}\rangle$, similarly for $S_{Y}$, and the cross spectrum is $S_{X Y}(\omega)=\frac{1}{2}\langle\{X(\omega), Y(-\omega)\}\rangle$. Since the cross spectrum of the two complex-valued frequencydomain quantities is usually not real, information may be lost in homodyne detection. This is represented in Fig. 1(a), which shows how positive and negative sideband frequencies sum up.

There has been little earlier discussion on the recovery of complex-valued squeezing correlations hidden to ordinary homodyne detection. Quantum squeezing in the hidden regime has been achieved in optics in one experiment via a modification of the two sidebands [46]. In the classical limit, an analogous noise reduction was recently obtained in a cavity optomechanical experiment [47] using digital filtering. Quite recently it was proposed [44] that the complex correlations could be accessed with a bichromatic LO, i.e., $\alpha_{0}(t)=\left|\alpha_{-}\right| \exp \left(-i \omega_{s} t-i \theta_{-}\right)+\left|\alpha_{+}\right| \exp \left(i \omega_{s} t-i \theta_{+}\right)$, as displayed in Fig. 1(b). The resulting noise spectrum can be written in a simple form at zero frequency (in the frequency frame oscillating at $\omega_{0}$ ) [48],

$$
\begin{aligned}
S_{X}^{\theta_{ \pm}}(0)= & \left|\alpha_{X}\right|^{2} \mathcal{C}_{11}\left(\omega_{s}\right)+\left|\alpha_{Y}\right|^{2} \mathcal{C}_{22}\left(\omega_{s}\right) \\
& +2 \operatorname{Re}\left[\alpha_{X}^{*} \alpha_{Y}^{*} \mathcal{C}_{12}\left(\omega_{s}\right)\right],
\end{aligned}
$$

where $\alpha_{X}=(1 / \sqrt{2})\left(\alpha_{+}+\alpha_{-}^{*}\right)$, and $\alpha_{Y}=(i / \sqrt{2})\left(\alpha_{+}^{*}-\alpha_{-}\right)$. We have denoted the respective spectra with the correlation matrix elements $\mathcal{C}_{i j}$, for example, $\mathcal{C}_{12} \equiv S_{X Y}$. The relation given by Eq. (2) allows us to interpret $S_{X}^{\theta_{ \pm}}(0)$ as a quadratic form in the variables $\alpha_{X}$ and $\alpha_{Y}$ associated with the matrix $\mathcal{C}_{i j}(\omega)$. The measurement of $S_{X}^{\theta_{ \pm}}(0)$ accesses the smallest (largest) eigenvalue of $\mathcal{C}_{i j}\left(\omega_{s}\right)$ by the choice of $\alpha_{X}, \alpha_{Y}$ in such a way that the vector $\left(\alpha_{X}, \alpha_{Y}\right)^{T}$ corresponds to the eigenvector associated to the smallest (largest) eigenvalue of $\mathcal{C}_{i j}\left(\omega_{s}\right)$, thereby revealing correlations hidden to homodyne detection.

The realization of the measurement leading to Eq. (2) is not restricted to a specific system. We choose to work in a generic cavity optomechanical setup [44], where the observability of the interesting quantities is expected to be well within reach. The interaction between the electromagnetic
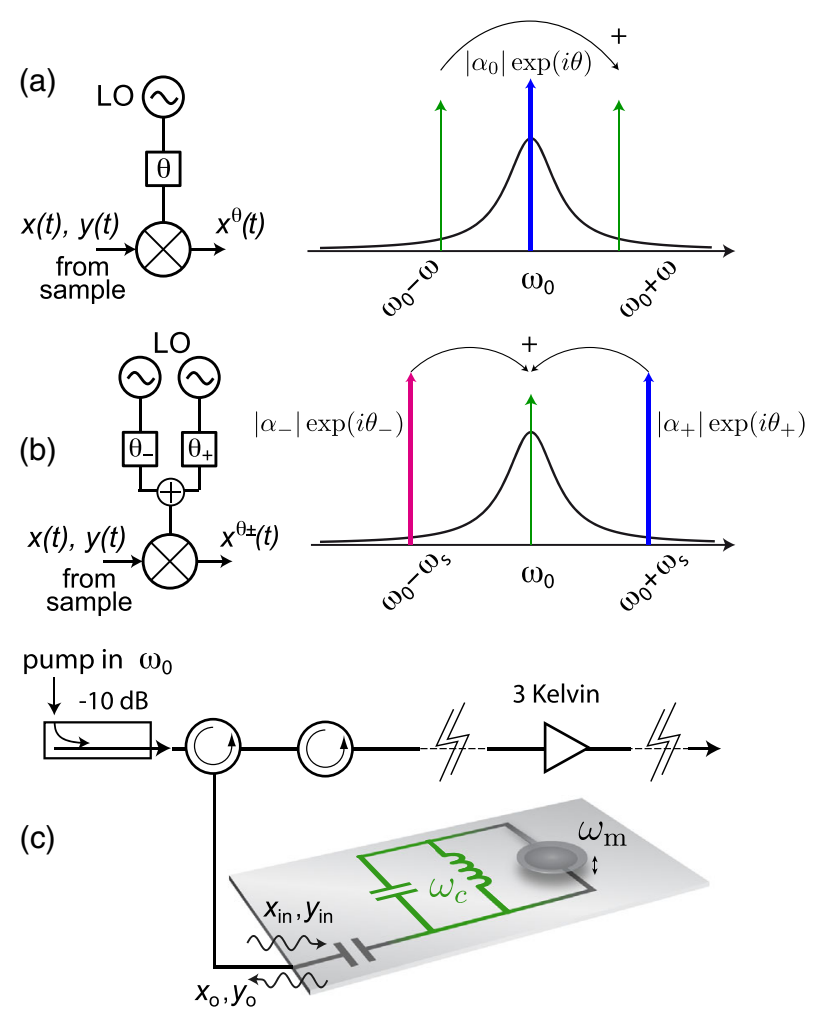

FIG. 1. Detection of squeezing correlations. The signal is injected into a mixer that multiplies it with LO waveforms. (a) In the case of standard homodyne detection, the LO is a single sinusoid with frequency $\omega_{0}$ and phase $\theta$, coinciding with the frequency of the pump used to create correlations in the resonator. This essentially sums up negative and positive frequencies $\pm \omega$ with respect to $\omega_{0}$. (b) Bichromatic LO with two phases can detect complex quantum correlations that may be invisible in homodyne detection. (c) Sketch of the electromechanical experiment, where ponderomotive squeezing of microwaves is created in a sample comprising a micromechanical oscillator parametrically coupled to a microwave resonator.

cavity (frequency $\omega_{c}$, damping rate $\kappa$, and the field operators $a^{\dagger}, a$ ), and the mechanical oscillator (frequency $\omega_{m}$, damping rate $\gamma_{m}$, operators $\left.b^{\dagger}, b\right)$ is of the form $g_{0} a^{\dagger} a\left(b^{\dagger}+b\right)$, where the single-photon coupling $g_{0} \ll \kappa$ is the small parameter. In order to obtain an effectively strong electromechanical coupling, a strong sinusoidal pump tone at frequency $\omega_{0} \simeq \omega_{c}$ is injected in the system. One can write the pump frequency using the detuning $\Delta \equiv \omega_{0}-\omega_{c}$. The pump induces a photon number $n_{c}$ in the cavity, and consequently one obtains a linearized interaction $G\left(a^{\dagger}+a\right)\left(b^{\dagger}+b\right)$ with the effective coupling $G=g_{0} \sqrt{n_{c}} \gg g_{0}$.

The dynamics is commonly written by the use of inputoutput theory of optical cavities for the linearized system. There is incoming electromagnetic noise at the device input, which in the present case of low temperature $k_{B} T \ll$ $\hbar \omega_{c}$ is composed of vacuum noise having the quadratures $x_{\text {in }}(t)$ and $y_{\text {in }}(t)$. The mechanical oscillator phonon number $n_{m}^{T} \simeq k_{B} T / \hbar \omega_{m}$, on the other hand, is relatively far from the 
ground state. The field quadratures leaking out from the cavity receive a contribution by the classical dynamics in the electromechanical system, but also by the fundamental measurement quantum backaction. In order to obtain the output field $X_{\mathrm{o}}(t), Y_{\mathrm{o}}(t)$, for simplicity we do not in the following write down the mechanical thermal noise. The frequency-domain quadratures are [48]

$$
\begin{aligned}
& X_{\mathrm{o}}(\omega)=\mathcal{A}_{X X} X_{\text {in }}(\omega)+\mathcal{A}_{X Y} Y_{\text {in }}(\omega), \\
& Y_{\mathrm{o}}(\omega)=\mathcal{A}_{Y X} X_{\text {in }}(\omega)+\mathcal{A}_{Y Y} Y_{\text {in }}(\omega),
\end{aligned}
$$

where the coefficients are

$$
\begin{aligned}
& \mathcal{A}_{X X}=\kappa \eta \chi_{\mathrm{c}}\left(\frac{\kappa}{2}-i \omega\right)-1, \quad \mathcal{A}_{X Y}=\kappa \eta \chi_{\mathrm{c}} \Delta, \\
& \mathcal{A}_{Y X}=-\kappa \eta \chi_{\mathrm{c}}\left(\Delta+4 G^{2} \omega_{m} \chi_{m}\right), \\
& \mathcal{A}_{Y Y}=\kappa \eta \chi_{\mathrm{c}}\left(\frac{\kappa}{2}-i \omega\right)-1,
\end{aligned}
$$

and $\eta=\left[1+4 G \omega_{m} \chi_{m} \Delta \chi_{\mathrm{c}}\right]^{-1}, \quad \chi_{\mathrm{c}}=\left\{[(\kappa / 2)-i \omega]^{2}+\Delta^{2}\right\}^{-1}$, $\chi_{m}=\left\{\left[\left(\gamma_{m} / 2\right)-i \omega\right]^{2}+\omega_{m}^{2}\right\}^{-1}$.

Ponderomotive squeezing qualitatively arises because the measurement backaction affects each of the output quadratures in a distinct way. The case $\Delta=0$ represents the most direct example, with $A_{X X}=A_{Y Y}$ and $A_{X Y}=0$, but $A_{Y X} \neq 0$. This, on one hand, implies squeezing; $\left\langle X_{\mathrm{o}}(\omega) X_{\mathrm{o}}(-\omega)\right\rangle \neq\left\langle Y_{\mathrm{o}}(\omega) Y_{\mathrm{o}}(-\omega)\right\rangle$ and, on the other hand, the appearance of nontrivial correlations among quadratures; $\left\langle X_{\mathrm{o}}(\omega) Y_{\mathrm{o}}(-\omega)\right\rangle \neq 0$. The emergence of nontrivial correlations is related to the fact that it is not possible to recast Eqs. (3a) and (3b) in diagonal form through a pair of orthogonal transformations of the input and output quadratures. Another example of a system for which Eqs. (3a) and (3b) cannot be written in diagonal form, hence leading to a mixing of the quadrature signals and a complex-valued $\mathcal{C}_{12}(\omega)$, is represented by a phase-mixing amplification (PMA) setup [43,49], showing how PMA and hidden correlations are closely related concepts.

Our experimental scheme is that of microwave cavity optomechanics; see Fig. 1(c). We use a superconducting on-chip cavity resonator (frequency $\omega_{c} / 2 \pi \simeq 7.31 \mathrm{GHz}$ ) coupled to a mechanical drum oscillator that has the frequency $\omega_{m} / 2 \pi \simeq 9.204 \mathrm{MHz}$ and the damping rate $\gamma_{m} / 2 \pi \simeq 120 \mathrm{~Hz}$. The single-sided cavity is strongly coupled to the measurement port through the coupling rate $\kappa_{E} / 2 \pi \simeq 27.7 \mathrm{MHz}$. The cavity also has internal losses at the rate $\kappa_{I} / 2 \pi \simeq 100 \mathrm{kHz}$, and the cavity losses sum up to $\kappa=\kappa_{I}+\kappa_{E} \simeq 2 \pi \times 27.8 \mathrm{MHz}$. The parameters are selected such that we operate somewhat in the bad-cavity limit $\kappa \gg \omega_{m}$ and the cavity responds fast to the mechanical fluctuations induced by the incoming shot noise, and hence the amount of squeezing is optimized.
The output signal from the sample is directed via isolators and superconducting cables towards the amplifier at 3 Kelvin. To avoid saturating the amplifier, we cancel the strong pump tone by summing up the original signal applied via a $-20 \mathrm{~dB}$ directional coupler, similar to our earlier works $[42,43,50,51]$. We detect the squeezing in the plane immediately preceding the $3 \mathrm{~K}$ amplifier. This is a regular phase-preserving high-electron-mobility transistor (HEMT) amplifier, and adds around $N_{\text {HEMT }} \approx 10$ quanta of noise to the signal. At room temperature, the signal is further amplified and digitized in a signal analyzer that provides the in-phase and out-of-phase quadratures.

Squeezing can be quantified as the noise in one quadrature $N^{\theta}$ in units of the vacuum noise in that quadrature, $N_{z p}^{\theta}=\frac{1}{4}$,

$$
\mathcal{S}=\frac{N^{\theta}}{N_{z p}^{\theta}} ;
$$

therefore, $\mathcal{S}<1$ (or $0 \mathrm{~dB}$ ) entails quantum squeezing. Squeezed microwaves have to be detected inside the refrigerator, because thermal noise at room temperature would overwhelm the squeezing. To infer the squeezing, we measure the quadrature spectral density $S^{\theta}$ with the pump tone on, and in a separate measurement $S_{\text {off }}^{\theta}$ with the pump tone off. This allows us to use $N_{\text {HEMT }}$ as a reference, which remains unchanged in both measurements, and was calibrated against a tunable noise source in a separate cooldown using the procedure of Ref. [43]. For details, see [48].

When using a single LO at the pump frequency [regular homodyne detection, Fig. 1(a)] we observe a strong phase dependence in the output noise. At LO phase values around $\theta \simeq \pi / 2$, we observe a maximum quantum squeezing of $1.1 \pm 0.4 \mathrm{~dB}$; see Fig. 2. The theoretical model shows a good agreement. For the fits, we used as free parameters the mechanical and cavity noise temperatures. The effective coupling and pump detuning are within $\simeq 5 \%$ of values calibrated via sideband cooling. Away from the mechanical resonance frequency we observe some excess noise $S>0 \mathrm{~dB}$ due to technical heating of the cavity.

To proceed towards detecting the hidden quantum correlations, we next explore the possible values of the spectrum $\mathcal{S}$ at a given frequency. We make a dense scan of the LO phase [Fig. 3(a)], and record at each frequency the minimum and maximum values of the spectrum. As displayed in Fig. 3(b), at the mechanical resonance frequency the minimum envelope develops a peak, which does not exhibit squeezing. In the figure we have also plotted the theoretically expected eigenvalues of the correlation matrix, and one can see that the mentioned peak clearly rises higher than the smaller eigenvalue.

We now discuss the main result obtained using the bichromatic LO [Fig. 1(b)]. The two LOs have frequencies 


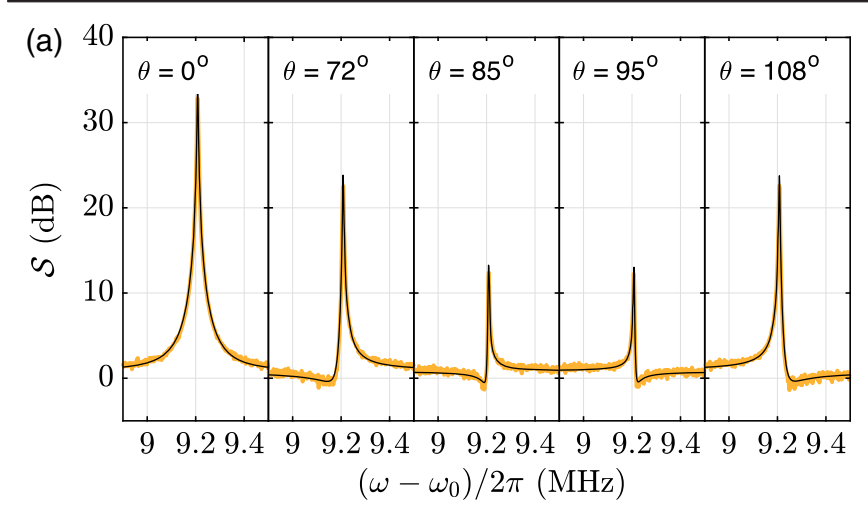

(b)

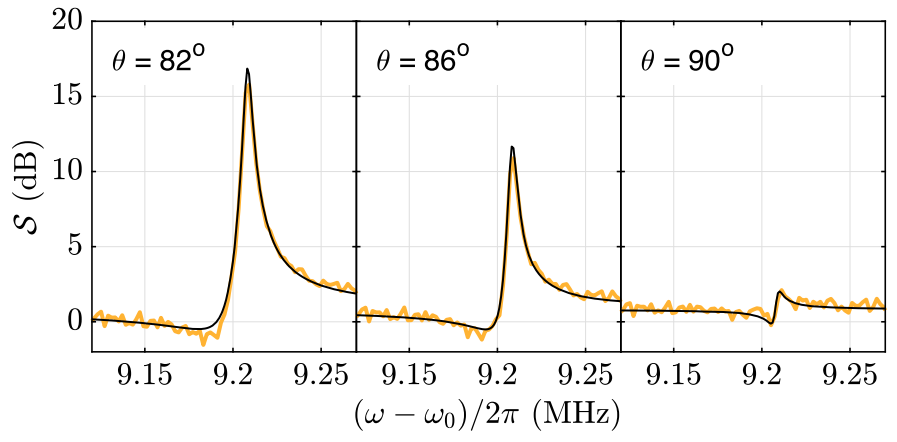

FIG. 2. Ponderomotive squeezing of microwaves. (a) Homodyne spectrum, Eq. (5), as referred to the input of the cryogenic amplifier. The phases of the sinusoidal local oscillator are written in the panels. (b) Detailed views of the phases around $\pi / 2$ that display the quantum squeezing. The thin black lines are theoretical fits. The experimental parameters are $G / 2 \pi \simeq 728 \mathrm{kHz}, n_{m}^{T} \simeq 517, n_{c}^{T} \simeq 0.07$, $\Delta / 2 \pi \simeq-620 \mathrm{kHz}$.

$\pm \omega_{s} \simeq \pm \omega_{m}$ symmetrically at both sides of the pump tone. In the general case they have a small detuning from the mechanical sideband, allowing us to map the frequency dependence of the correlation matrix; see Eq. (2). We create the two LOs digitally and optimize their amplitude and phase to maximize the squeezing around zero frequency. The optimized amplitude ratio of $\alpha_{-}$and $\alpha_{+}$differs only $0.2 \%$ from that predicted by the model. In Fig. 4 we display the results at different LO detuning values. With the complex detection, we recover squeezing around the zero frequency, which in the lab frame corresponds to the mechanical sidebands of the cavity resonance. The theoretical prediction, using the same parameters as in Fig. 2,

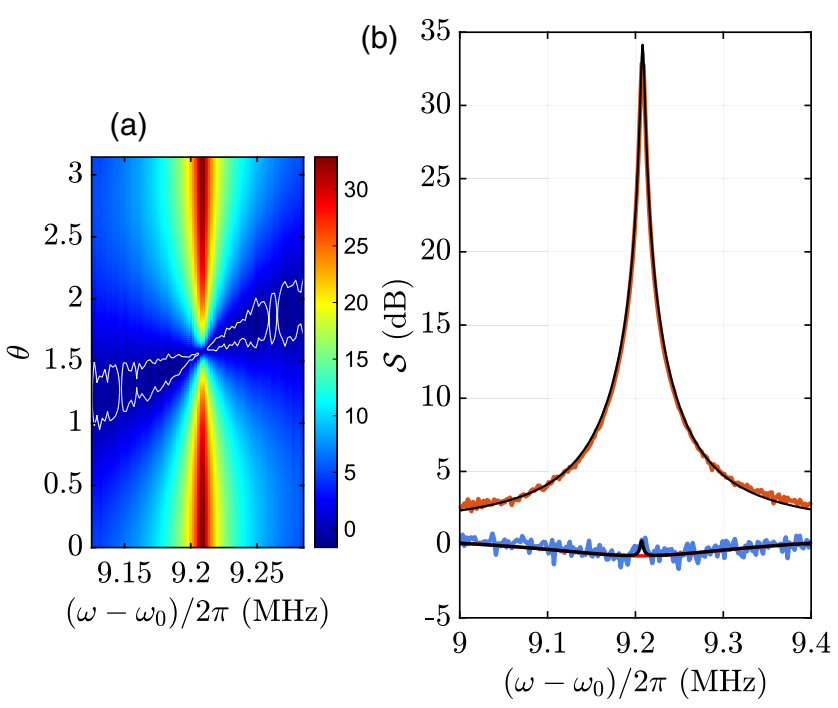

FIG. 3. Squeezing eigenvalues. (a) Homodyne noise spectrum shown as a color map. We observe quantum squeezing (value below $0 \mathrm{~dB}$ ) inside the white contours. (b) Maximum (red) and minimum (blue) envelope of the spectrum with respect to the local oscillator phase $\theta$ from the data in (a). The black lines are theory predictions, and the red theory line is the lower eigenvalue of the correlation matrix. accurately follows the data. Finally, in Fig. 5 we present the squeezing around zero frequency as a function of bichromatic LO detuning, showing how we can map the eigenvalues of the correlation matrix within the "forbidden" region of $\sim \pm 5 \mathrm{kHz}$ around the mechanical resonance. While at small detunings the error bars grow larger (since there are only a few data points to consider in the spectra of Fig. 4); several points are around 2 standard deviations below the expected result with ideal homodyne detection (black line), demonstrating that the bichromatic LO provides a significant improvement over homodyne detection. We note that the average value does not quite reach that of

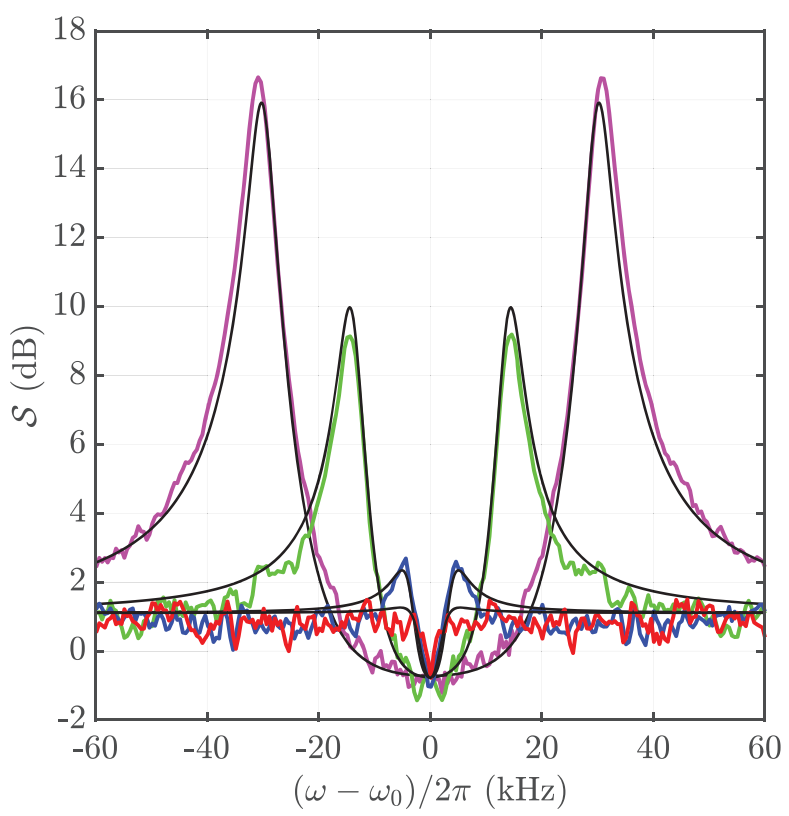

FIG. 4. Complex squeezing spectrum. The detuning of the two LOs are varied between $\left(\omega_{s}-\omega_{m}\right) / 2 \pi=[-31,-15,-4.5,1] \mathrm{kHz}$ from top to bottom (magenta, green, blue, red). The phase is optimized in order to minimize the noise for each detuning value. Black lines are theoretical fits. 


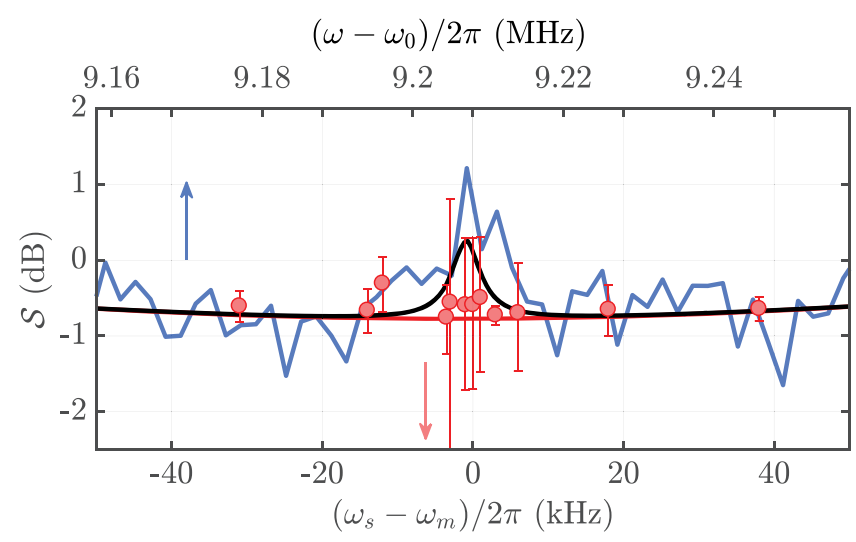

FIG. 5. Mapping the correlation matrix. The solid symbols, with $2 \sigma$ error bars, denote the minimum of the complex squeezing spectrum at zero frequency as a function of the bi-LO detuning. This is compared to the theoretical prediction for the smallest eigenvalues of the correlation matrix (red line). The hidden quantum correlations are given by the data points falling below the lower homodyne envelope (blue trace) and the corresponding theory line (black), around zero detuning. The frequency axis for the homodyne data is given by the upper labels.

the homodyne detection, which is likely because the LOs cannot be well optimized under strong scatter of the data.

To conclude, we have investigated propagating microwaves to recover quantum correlations that hitherto have remained elusive. Our work also confirms ponderomotive squeezing at a frequency range 4 orders of magnitude lower than previously demonstrated. The hidden correlations are foreseen to exist and be measurable also in other systems where the output field has to be expressed as a mixture of the quadratures of the field entering the system. In the present system, we expect the reduced noise at a resonant condition to be useful for sensitive force detection.

This work was supported by the Academy of Finland (Contracts No. 250280, No. 308290, No. 307757, No. 312296, and No. 275245), by the European Research Council (Grant No. 615755-CAVITYQPD), and by the Centre for Quantum Engineering at Aalto University. We acknowledge funding from the European Union's Horizon 2020 research and innovation program under Grant No. 732894 (FETPRO HOT). We acknowledge the facilities and technical support of Otaniemi research infrastructure for Micro and Nanotechnologies (OtaNano).

*mika.sillanpaa@aalto.fi

[1] D. F. Walls, Squeezed states of light, Nature (London) 306, 141 (1983).

[2] R. Loudon and P. L. Knight, Squeezed light, J. Mod. Opt. 34, 709 (1987).

[3] E. S. Polzik, J. Carri, and H. J. Kimble, Spectroscopy with Squeezed Light, Phys. Rev. Lett. 68, 3020 (1992).
[4] S. Steinlechner, J. Bauchrowitz, M. Meinders, H. MüllerEbhardt, K. Danzmann, and R. Schnabel, Quantum-dense metrology, Nat. Photonics 7, 626 (2013).

[5] M. A. Taylor, J. Janousek, V. Daria, J. Knittel, B. Hage, H.-A. Bachor, and W. P. Bowen, Biological measurement beyond the quantum limit, Nat. Photonics 7, 229 (2013).

[6] C. Schäfermeier, H. Kerdoncuff, U. B. Hoff, H. Fu, A. Huck, J. Bilek, G. I. Harris, W. P. Bowen, T. Gehring, and U.L. Andersen, Quantum enhanced feedback cooling of a mechanical oscillator using nonclassical light, Nat. Commun. 7, 13628 (2016).

[7] N. S. Kampel, R. W. Peterson, R. Fischer, P.-L. Yu, K. Cicak, R. W. Simmonds, K. W. Lehnert, and C. A. Regal, Improving Broadband Displacement Detection with Quantum Correlations, Phys. Rev. X 7, 021008 (2017).

[8] N. Didier, A. Kamal, W. D. Oliver, A. Blais, and A. A. Clerk, Heisenberg-Limited Qubit Read-Out with Two-Mode Squeezed Light, Phys. Rev. Lett. 115, 093604 (2015).

[9] D. M. Toyli, A. W. Eddins, S. Boutin, S. Puri, D. Hover, V. Bolkhovsky, W. D. Oliver, A. Blais, and I. Siddiqi, Resonance Fluorescence from an Artificial Atom in Squeezed Vacuum, Phys. Rev. X 6, 031004 (2016).

[10] A. Bienfait, P. Campagne-Ibarcq, A. H. Kiilerich, X. Zhou, S. Probst, J. J. Pla, T. Schenkel, D. Vion, D. Esteve, J. J. L. Morton, K. Moelmer, and P. Bertet, Magnetic Resonance with Squeezed Microwaves, Phys. Rev. X 7, 041011 (2017).

[11] J. Aasi, J. Abadie, B. P. Abbott, R. Abbott, T. D. Abbott, M. R. Abernathy, C. Adams, T. Adams, and P. Addesso et al., Enhanced sensitivity of the LIGO gravitational wave detector by using squeezed states of light, Nat. Photonics 7, 613 (2013).

[12] R. Schnabel, Squeezed states of light and their applications in laser interferometers, Phys. Rep. 684, 1 (2017).

[13] A. Furusawa, J. L. Sørensen, S. L. Braunstein, C. A. Fuchs, H. J. Kimble, and E.S. Polzik, Unconditional quantum teleportation, Science 282, 706 (1998).

[14] S. L. Braunstein and P. van Loock, Quantum information with continuous variables, Rev. Mod. Phys. 77, 513 (2005).

[15] C. Weedbrook, S. Pirandola, R. García-Patrón, N. J. Cerf, T. C. Ralph, J. H. Shapiro, and S. Lloyd, Gaussian quantum information, Rev. Mod. Phys. 84, 621 (2012).

[16] K. W. Murch, S. J. Weber, K. M. Beck, E. Ginossar, and I. Siddiqi, Reduction of the radiative decay of atomic coherence in squeezed vacuum, Nature (London) 499, 62 (2013).

[17] A. Motazedifard, F. Bemani, M. H. Naderi, R. Roknizadeh, and D. Vitali, Force sensing based on coherent quantum noise cancellation in a hybrid optomechanical cavity with squeezed-vacuum injection, New J. Phys. 18, 073040 (2016).

[18] J. B. Clark, F. Lecocq, R. W. Simmonds, J. Aumentado, and J. D. Teufel, Sideband cooling beyond the quantum backaction limit with squeezed light, Nature (London) 541, 191 (2017).

[19] R. E. Slusher, L. W. Hollberg, B. Yurke, J. C. Mertz, and J. F. Valley, Observation of Squeezed States Generated by Four-Wave Mixing in an Optical Cavity, Phys. Rev. Lett. 55, 2409 (1985).

[20] R. M. Shelby, M. D. Levenson, S. H. Perlmutter, R. G. DeVoe, and D.F. Walls, Broad-Band Parametric 
Deamplification of Quantum Noise in an Optical Fiber, Phys. Rev. Lett. 57, 691 (1986).

[21] L.-A. Wu, H. J. Kimble, J. L. Hall, and H. Wu, Generation of Squeezed States by Parametric Down Conversion, Phys. Rev. Lett. 57, 2520 (1986).

[22] A. Heidmann, R. J. Horowicz, S. Reynaud, E. Giacobino, C. Fabre, and G. Camy, Observation of Quantum Noise Reduction on Twin Laser Beams, Phys. Rev. Lett. 59, 2555 (1987).

[23] L.-A. Wu, M. Xiao, and H. J. Kimble, Squeezed states of light from an optical parametric oscillator, J. Opt. Soc. Am. B 4, 1465 (1987).

[24] B. Yurke, P. Kaminsky, R. Miller, E. Whittaker, A. Smith, A. Silver, and R. Simon, Observation of 4.2-K EquilibriumNoise Squeezing via a Josephson-Parametric Amplifier, Phys. Rev. Lett. 60, 764 (1988).

[25] R. Movshovich, B. Yurke, P. G. Kaminsky, A. D. Smith, A. H. Silver, R. W. Simon, and M. V. Schneider, Observation of Zero-Point Noise Squeezing via a Josephson-Parametric Amplifier, Phys. Rev. Lett. 65, 1419 (1990).

[26] M. A. Castellanos-Beltran, K. D. Irwin, G. C. Hilton, L. R. Vale, and K. W. Lehnert, Amplification and squeezing of quantum noise with a tunable Josephson metamaterial, Nat. Phys. 4, 929 (2008).

[27] C. Eichler, D. Bozyigit, C. Lang, M. Baur, L. Steffen, J. M. Fink, S. Filipp, and A. Wallraff, Observation of Two-Mode Squeezing in the Microwave Frequency Domain, Phys. Rev. Lett. 107, 113601 (2011).

[28] F. Mallet, M. A. Castellanos-Beltran, H. S. Ku, S. Glancy, E. Knill, K. D. Irwin, G. C. Hilton, L. R. Vale, and K. W. Lehnert, Quantum State Tomography of an Itinerant Squeezed Microwave Field, Phys. Rev. Lett. 106, 220502 (2011).

[29] L. Zhong, E. P. Menzel, R. Di Candia, P. Eder, M. Ihmig, A. Baust, M. Haeberlein, E. Hoffmann, K. Inomata, T. Yamamoto, Y. Nakamura, E. Solano, F. Deppe, A. Marx, and R. Gross, Squeezing with a flux-driven Josephson parametric amplifier, New J. Phys. 15, 125013 (2013).

[30] E. Flurin, N. Roch, F. Mallet, M. H. Devoret, and B. Huard, Generating Entangled Microwave Radiation Over Two Transmission Lines, Phys. Rev. Lett. 109, 183901 (2012).

[31] K. G. Fedorov, L. Zhong, S. Pogorzalek, P. Eder, M. Fischer, J. Goetz, E. Xie, F. Wulschner, K. Inomata, T. Yamamoto, Y. Nakamura, R. Di Candia, U. Las Heras, M. Sanz, E. Solano, E. P. Menzel, F. Deppe, A. Marx, and R. Gross, Displacement of Propagating Squeezed Microwave States, Phys. Rev. Lett. 117, 020502 (2016).

[32] S. Mancini and P. Tombesi, Quantum-noise reduction by radiation pressure, Phys. Rev. A 49, 4055 (1994).

[33] C. Fabre, M. Pinard, S. Bourzeix, A. Heidmann, E. Giacobino, and S. Reynaud, Quantum-noise reduction using a cavity with a movable mirror, Phys. Rev. A 49, 1337 (1994).

[34] D. W. C. Brooks, T. Botter, S. Schreppler, T. P. Purdy, N. Brahms, and D. M. Stamper-Kurn, Nonclassical light generated by quantum-noise-driven cavity optomechanics, Nature (London) 488, 476 (2012).

[35] A. H. Safavi-Naeini, S. Gröblacher, J. T. Hill, J. Chan, M. Aspelmeyer, and O. Painter, Squeezed light from a silicon micromechanical resonator, Nature (London) 500, 185 (2013).
[36] T. P. Purdy, P.-L. Yu, R. W. Peterson, N. S. Kampel, and C. A. Regal, Strong Optomechanical Squeezing of Light, Phys. Rev. X 3, 031012 (2013).

[37] W. H. Padkær Nielsen, Y. Tsaturyan, C. Bo Møller, E. S. Polzik, and A. Schliesser, Multimode optomechanical system in the quantum regime, Proc. Natl. Acad. Sci. U.S.A. 114, 62 (2017).

[38] T. P. Purdy, K. E. Grutter, K. Srinivasan, and J. M. Taylor, Quantum correlations from a room-temperature optomechanical cavity, Science 356, 1265 (2017).

[39] V. Sudhir, R. Schilling, S. A. Fedorov, H. Schütz, D. J. Wilson, and T.J. Kippenberg, Quantum Correlations of Light from a Room-Temperature Mechanical Oscillator, Phys. Rev. X 7, 031055 (2017).

[40] J. Suh, A. J. Weinstein, C. U. Lei, E. E. Wollman, S. K. Steinke, P. Meystre, A. A. Clerk, and K. C. Schwab, Mechanically detecting and avoiding the quantum fluctuations of a microwave field, Science 344, 1262 (2014).

[41] J. D. Teufel, F. Lecocq, and R. W. Simmonds, Overwhelming Thermomechanical Motion with Microwave Radiation Pressure Shot Noise, Phys. Rev. Lett. 116, 013602 (2016).

[42] C. F. Ockeloen-Korppi, E. Damskägg, J.-M. Pirkkalainen, A. A. Clerk, M. J. Woolley, and M. A. Sillanpää, Quantum Backaction Evading Measurement of Collective Mechanical Modes, Phys. Rev. Lett. 117, 140401 (2016).

[43] C. F. Ockeloen-Korppi, E. Damskägg, J.-M. Pirkkalainen, T. T. Heikkilä, F. Massel, and M. A. Sillanpää, Noiseless Quantum Measurement and Squeezing of Microwave Fields Utilizing Mechanical Vibrations, Phys. Rev. Lett. 118, 103601 (2017).

[44] L. F. Buchmann, S. Schreppler, J. Kohler, N. Spethmann, and D. M. Stamper-Kurn, Complex Squeezing and Force Measurement Beyond the Standard Quantum Limit, Phys. Rev. Lett. 117, 030801 (2016).

[45] R. J. Glauber, The quantum theory of optical coherence, Phys. Rev. 130, 2529 (1963).

[46] F. A. S. Barbosa, A. S. Coelho, K. N. Cassemiro, P. Nussenzveig, C. Fabre, M. Martinelli, and A.S. Villar, Beyond Spectral Homodyne Detection: Complete Quantum Measurement of Spectral Modes of Light, Phys. Rev. Lett. 111, 200402 (2013).

[47] A. Pontin, J. E. Lang, A. Chowdhury, P. Vezio, F. Marino, B. Morana, E. Serra, F. Marin, and T. S. Monteiro, Imaging Correlations in Heterodyne Spectra for Quantum Displacement Sensing, Phys. Rev. Lett. 120, 020503 (2018).

[48] See Supplemental Material at http://link.aps.org/ supplemental/10.1103/PhysRevLett.121.243601 for experimental and theoretical details.

[49] C. F. Ockeloen-Korppi, T. T. Heikkilä, M. A. Sillanpää, and F. Massel, Theory of phase-mixing amplification in an optomechanical system, Quantum Sci. Technol. 2, 035002 (2017).

[50] J.-M. Pirkkalainen, E. Damskägg, M. Brandt, F. Massel, and M. A. Sillanpää, Squeezing of Quantum Noise of Motion in a Micromechanical Resonator, Phys. Rev. Lett. 115, 243601 (2015).

[51] C. F. Ockeloen-Korppi, E. Damskägg, J. M. Pirkkalainen, M. Asjad, A. A. Clerk, F. Massel, M. J. Woolley, and M. A. Sillanpää, Stabilized entanglement of massive mechanical oscillators, Nature (London) 556, 478 (2018). 


\section{Revealing hidden quantum correlations in an electromechanical measurement - Supplementary material}

C. F. Ockeloen-Korppi, ${ }^{1}$ E. Damskägg, ${ }^{1}$ G. S. Paraoanu,${ }^{1}$ F. Massel,${ }^{2}$ and M. A. Sillanpää ${ }^{1, *}$

${ }^{1}$ Department of Applied Physics, Aalto University, P.O. Box 15100, FI-00076 AALTO, Finland

${ }^{2}$ Department of Physics and Nanoscience Center, University of Jyväskylä, P.O. Box 35 (YFL), FI-40014 University of Jyväskylä, Finland 


\section{DERIVATION OF THE OUTPUT FIELD CORRELATORS}

We derive here the equations of motion (EOMs) for the field and the mechanical quadratures generated by the linearized optomechanical Hamiltonian, extending the result given in [1] to finite pump detuning from the cavity resonance and to finite mismatch between the bichromatic sideband frequency $\omega_{\mathrm{s}}$ and the mechanical oscillator frequency $\omega_{\mathrm{m}}$. In a frame rotating at the pump frequency $\omega_{\mathrm{p}}$ the linearized Hamiltonian can be written as

$$
H=\frac{\Delta}{2}\left(X^{2}+Y^{2}\right)+2 G X Q
$$

where $\Delta=\omega_{\mathrm{c}}-\omega_{\mathrm{p}}$ and we have defined $X=\left(a^{\dagger}+a\right) / \sqrt{2}, Y=i\left(a^{\dagger}-a\right) \sqrt{2}, Q=\left(b^{\dagger}+b\right) / \sqrt{2}, P=i\left(b^{\dagger}-b\right) / \sqrt{2}$.

The quantum Langevin equations associated with the Hamiltonian given in Eq. (S1) can be written in the frequency domain as

$$
\begin{aligned}
& \left(\frac{\kappa}{2}-i \omega\right) X=\Delta Y+\sqrt{\kappa} X_{\mathrm{in}} \\
& \left(\frac{\kappa}{2}-i \omega\right) Y=-\Delta X-2 G Q+\sqrt{\kappa} Y_{\mathrm{in}} \\
& \left(\frac{\gamma}{2}-i \omega\right) Q=\omega_{\mathrm{m}} P+\sqrt{\gamma} Q_{\mathrm{in}} \\
& \left(\frac{\gamma}{2}-i \omega\right) P=-\omega_{\mathrm{m}} Q-2 G X+\sqrt{\gamma} P_{\mathrm{in}}
\end{aligned}
$$

where all operators are evaluated at the frequency $\omega$, unless otherwise specified.

Solving Eqs. (S2a-S2d), we can express the relation between the input $\left(X_{\text {in }}, Y_{\text {in }}\right)$ and the output $\left(X_{\mathrm{o}}=\sqrt{\kappa} X-X_{\text {in }}\right.$, $\left.Y_{\mathrm{o}}=\sqrt{\kappa} Y-Y_{\mathrm{in}}\right)$ quadratures for the cavity field as

$$
\begin{aligned}
& X_{\mathrm{o}}=\mathcal{A}_{\mathrm{XX}} X_{\mathrm{in}}+\mathcal{A}_{\mathrm{XY}} Y_{\mathrm{in}}+\mathcal{A}_{\mathrm{Xq}} Q_{\mathrm{in}}+\mathcal{A}_{\mathrm{Xp}} P_{\mathrm{in}} \\
& Y_{\mathrm{o}}=\mathcal{A}_{Y X} X_{\mathrm{in}}+\mathcal{A}_{\mathrm{YY}} Y_{\mathrm{in}}+\mathcal{A}_{\mathrm{Yq}} Q_{\mathrm{in}}+\mathcal{A}_{\mathrm{Yp}} P_{\mathrm{in}}
\end{aligned}
$$

where we have defined

$$
\begin{array}{ll}
\mathcal{A}_{\mathrm{XX}}=\kappa \eta \chi_{\mathrm{c}}\left(\frac{\kappa}{2}-i \omega\right)-1, & \mathcal{A}_{\mathrm{XY}}=\kappa \eta \chi_{\mathrm{c}} \Delta, \\
\mathcal{A}_{\mathrm{YX}}=-\kappa \eta \chi_{\mathrm{c}}\left(\Delta+4 G^{2} \omega_{\mathrm{m}} \chi_{\mathrm{m}}\right), & \mathcal{A}_{\mathrm{YY}}=\kappa \eta \chi_{\mathrm{c}}\left(\frac{\kappa}{2}-i \omega\right)-1, \\
\mathcal{A}_{\mathrm{Xq}}=-2 \sqrt{\kappa \gamma} G \eta \chi_{\mathrm{c}} \chi_{\mathrm{m}} \Delta\left(\frac{\gamma}{2}-i \omega\right), & \mathcal{A}_{\mathrm{Xp}}=-2 \sqrt{\kappa \gamma} G \eta \chi_{\mathrm{c}} \chi_{\mathrm{m}} \Delta \omega_{\mathrm{m}}, \\
\mathcal{A}_{\mathrm{Yq}}=-2 \sqrt{\kappa \gamma} G \eta \chi_{\mathrm{c}} \chi_{\mathrm{m}}\left(\frac{\kappa}{2}-i \omega\right)\left(\frac{\gamma}{2}-i \omega\right), & \mathcal{A}_{\mathrm{Yp}}=-2 \sqrt{\kappa \gamma} G \eta \chi_{\mathrm{c}} \chi_{\mathrm{m}}\left(\frac{\kappa}{2}-i \omega\right) \omega_{\mathrm{m}} .
\end{array}
$$

with

$$
\chi_{\mathrm{c}}=\left[\left(\frac{\kappa}{2}-i \omega\right)^{2}+\Delta^{2}\right]^{-1}, \quad \chi_{\mathrm{m}}=\left[\left(\frac{\gamma}{2}-i \omega\right)^{2}+\omega_{\mathrm{m}}^{2}\right]^{-1}, \quad \eta=\left[1+4 G \omega_{\mathrm{m}} \chi_{\mathrm{m}} \Delta \chi_{\mathrm{c}}\right]^{-1} .
$$

From Eqs. (S3a, S3b), it is possible to evaluate the symmetrized covariance matrix for the output field correlator, which, owing to time translation invariance, becomes

$$
C_{\mathrm{ij}}(\omega)=\frac{1}{2}\left\langle\left\{X_{\mathrm{o}}^{\mathrm{i}}(\omega), X_{\mathrm{o}}^{\mathrm{j}}(-\omega)\right\}\right\rangle
$$

where $\mathrm{i}, \mathrm{j}=1,2\left(X_{\mathrm{o}}^{1}=X_{\mathrm{o}}, X_{\mathrm{o}}^{2}=Y_{\mathrm{o}}\right)$. Each element of $C_{\mathrm{ij}}(\omega)$ is written as the sum of a contribution originating from the mechanical thermal bath $\left(\langle\ldots\rangle_{\mathrm{m}}\right)$ and a contribution associated with the cavity thermal bath $\left(\langle\ldots\rangle_{\mathrm{c}}\right)$. Recognizing that, for each of the coefficients given in Eqs. (S4a-S4d) we have that $\mathcal{A}_{\mathrm{ij}}(-\omega)=\mathcal{A}_{\mathrm{ij}}^{*}(\omega)$, we can derive the following relations for the dependence of $C_{\mathrm{ij}}(\omega)$ on the bath field correlators

$$
\begin{aligned}
& \frac{1}{2}\left\langle\left\{X_{\mathrm{o}}(\omega), X_{\mathrm{o}}(-\omega)\right\}\right\rangle_{\mathrm{c}}=\left[\left|\mathcal{A}_{\mathrm{XX}}\right|^{2}+\left|\mathcal{A}_{\mathrm{XY}}\right|^{2}\right]\left(n_{\mathrm{c}}+\frac{1}{2}\right) \\
& \frac{1}{2}\left\langle\left\{Y_{\mathrm{O}}(\omega), Y_{\mathrm{o}}(-\omega)\right\}\right\rangle_{\mathrm{c}}=\left[\left|\mathcal{A}_{\mathrm{YX}}\right|^{2}+\left|\mathcal{A}_{\mathrm{YY}}\right|^{2}\right]\left(n_{\mathrm{c}}+\frac{1}{2}\right) \\
& \frac{1}{2}\left\langle\left\{X_{\mathrm{O}}(\omega), Y_{\mathrm{O}}(-\omega)\right\}\right\rangle_{\mathrm{c}}=\left[\mathcal{A}_{\mathrm{YX}} \mathcal{A}_{\mathrm{XX}}{ }^{*}+\mathcal{A}_{\mathrm{YY}} \mathcal{A}_{\mathrm{XY}}{ }^{*}\right]\left(n_{\mathrm{c}}+\frac{1}{2}\right),
\end{aligned}
$$


and on the mechanical field correlators

$$
\begin{aligned}
& \frac{1}{2}\left\langle\left\{X_{\mathrm{o}}(\omega), X_{\mathrm{o}}(-\omega)\right\}\right\rangle_{\mathrm{m}}=\left[\left|\mathcal{A}_{\mathrm{Xq}}\right|^{2}+\left|\mathcal{A}_{\mathrm{Xp}}\right|^{2}\right]\left(n_{\mathrm{m}}+\frac{1}{2}\right) \\
& \frac{1}{2}\left\langle\left\{Y_{\mathrm{o}}(\omega), Y_{\mathrm{o}}(-\omega)\right\}\right\rangle_{\mathrm{m}}=\left[\left|\mathcal{A}_{\mathrm{Yq}}\right|^{2}+\left|\mathcal{A}_{\mathrm{Yp}}\right|^{2}\right]\left(n_{\mathrm{m}}+\frac{1}{2}\right) \\
& \frac{1}{2}\left\langle\left\{X_{\mathrm{o}}(\omega), Y_{\mathrm{o}}(-\omega)\right\}\right\rangle_{\mathrm{m}}=\left[\mathcal{A}_{\mathrm{Yq}} \mathcal{A}_{\mathrm{Xq}}{ }^{*}+\mathcal{A}_{\mathrm{Yp}} \mathcal{A}_{\mathrm{Xp}}{ }^{*}\right]\left(n_{\mathrm{m}}+\frac{1}{2}\right) .
\end{aligned}
$$

Eqs. (S7a, S7c) can be explicitly written as

$$
\begin{aligned}
& \frac{1}{2}\left\langle\left\{X_{\mathrm{o}}(\omega), X_{\mathrm{o}}(-\omega)\right\}\right\rangle_{\mathrm{c}}=\kappa\left\{\kappa|\eta|^{2}\left|\chi_{\mathrm{c}}\right|^{2}\left(\frac{\kappa^{2}}{4}+\omega^{2}+\Delta^{2}\right)-2 \operatorname{Re}\left[\eta \chi_{\mathrm{c}}\left(\frac{\kappa}{2}-i \omega\right)+1\right]\right\}\left(n_{\mathrm{c}}+\frac{1}{2}\right) \\
& \frac{1}{2}\left\langle\left\{Y_{\mathrm{o}}(\omega), Y_{\mathrm{o}}(-\omega)\right\}\right\rangle_{\mathrm{c}}=\kappa\left\{\kappa|\eta|^{2}\left|\chi_{\mathrm{c}}\right|^{2}\left(\frac{\kappa^{2}}{4}+\omega^{2}+\left|\Delta+4 G^{2} \omega_{\mathrm{m}} \chi_{\mathrm{m}}\right|^{2}\right)\right. \\
& \left.-2 \operatorname{Re}\left[\eta \chi_{\mathrm{c}}\left(\frac{\kappa}{2}-i \omega\right)+1\right]\right\}\left(n_{\mathrm{c}}+\frac{1}{2}\right) \\
& \frac{1}{2}\left\langle\left\{X_{\mathrm{o}}(\omega), Y_{\mathrm{o}}(-\omega)\right\}\right\rangle_{\mathrm{c}}=\kappa\left\{\kappa|\eta|^{2}\left|\chi_{\mathrm{c}}\right|^{2}\left[2 \kappa G^{2} \omega_{\mathrm{m}}+2 i \omega\left(\Delta+2 G^{2} \omega_{\mathrm{m}} \chi_{\mathrm{m}}\right)\right]\right. \\
& \left.+\eta \chi_{\mathrm{c}}\left(\Delta+4 G^{2} \omega_{\mathrm{m}} \chi_{\mathrm{m}}\right)-\Delta \eta^{*} \chi_{\mathrm{c}}^{*}\right\}\left(n_{\mathrm{c}}+\frac{1}{2}\right)
\end{aligned}
$$

and Eqs. (S8a, S8c) become

$$
\begin{aligned}
& \frac{1}{2}\left\langle\left\{X_{\mathrm{o}}(\omega), X_{\mathrm{o}}(-\omega)\right\}\right\rangle_{\mathrm{m}}=2 \kappa \gamma|\eta| G^{2} \Delta^{2}\left|\chi_{\mathrm{c}}\right|^{2}\left|\chi_{\mathrm{m}}\right|^{2}\left(\gamma^{2} / 4-\omega^{2}+\omega_{\mathrm{m}}^{2}\right)\left(n_{\mathrm{m}}+\frac{1}{2}\right) \\
& \frac{1}{2}\left\langle\left\{Y_{\mathrm{o}}(\omega), Y_{\mathrm{o}}(-\omega)\right\}\right\rangle_{\mathrm{m}}=2 \kappa \gamma|\eta|^{2} G^{2}\left(\kappa^{2} / 4+\omega^{2}\right)\left|\chi_{\mathrm{c}}\right|^{2}\left|\chi_{\mathrm{m}}\right|^{2}\left(\gamma^{2} / 4-\omega^{2}+\omega_{\mathrm{m}}^{2}\right)\left(n_{\mathrm{m}}+\frac{1}{2}\right) \\
& \frac{1}{2}\left\langle\left\{X_{\mathrm{o}}(\omega), Y_{\mathrm{o}}(-\omega)\right\}\right\rangle_{\mathrm{m}}=2 \kappa \gamma|\eta| G^{2} \Delta \kappa\left|\chi_{\mathrm{c}}\right|^{2}\left|\chi_{\mathrm{m}}\right|^{2}\left(\gamma^{2} / 4-\omega^{2}+\omega_{\mathrm{m}}^{2}\right)\left(n_{\mathrm{m}}+\frac{1}{2}\right)
\end{aligned}
$$

\section{COMPLEX DETECTION AND COVARIANCE MATRIX}

In order to extract all information encoded in the covariance matrix, we consider the power spectrum resulting from the bichromatic detection:

$$
S_{\mathrm{bi}}(\omega)=\frac{1}{2}\langle\{\Sigma(\omega), \Sigma(-\omega)\}\rangle
$$

where

$$
\Sigma(\omega)=\frac{1}{\sqrt{2}}\left[\alpha_{\mathrm{X}} X_{\mathrm{o}}\left(\omega-\omega_{s}\right)+\alpha_{\mathrm{X}}^{*} X_{\mathrm{o}}\left(\omega+\omega_{s}\right)+\alpha_{\mathrm{Y}} Y_{\mathrm{o}}\left(\omega-\omega_{s}\right)+\alpha_{\mathrm{Y}}^{*} Y_{\mathrm{o}}\left(\omega+\omega_{s}\right)\right]
$$

where $\omega_{\mathrm{s}}$ is the detuning of the LO frequency from the pump tone. $S_{\mathrm{bi}}(\omega)$ can be written in terms of the covariance matrix $C_{\mathrm{ij}}(\omega)$ as

$$
\begin{aligned}
S_{\mathrm{bi}}(\omega)= & \frac{\left|\alpha_{\mathrm{X}}\right|^{2}}{2}\left[C_{11}\left(\omega-\omega_{\mathrm{s}}\right)+C_{11}\left(\omega+\omega_{\mathrm{s}}\right)\right]+\frac{\left|\alpha_{\mathrm{Y}}\right|^{2}}{2}\left[C_{22}\left(\omega-\omega_{\mathrm{s}}\right)+C_{22}\left(\omega+\omega_{\mathrm{s}}\right)\right] \\
& +\operatorname{Re}\left[\alpha_{\mathrm{A}} \alpha_{\mathrm{Y}} C_{12}\left(\omega-\omega_{\mathrm{s}}\right)+\alpha_{\mathrm{X}}^{*} \alpha_{\mathrm{Y}}^{*} C_{12}\left(\omega+\omega_{\mathrm{s}}\right)\right]
\end{aligned}
$$

which for $\omega=0$ and $\omega_{\mathrm{s}}=\omega_{\mathrm{m}}$, due to the frequency symmetry of $C_{\mathrm{ij}}(\omega)$, can be written as

$$
\begin{aligned}
S_{\mathrm{bi}}(0)= & \left|\alpha_{\mathrm{X}}\right|^{2} C_{11}\left(\omega_{\mathrm{s}}\right)+\left|\alpha_{\mathrm{Y}}\right|^{2} C_{22}\left(\omega_{\mathrm{s}}\right) \\
& +2 \operatorname{Re}\left[\alpha_{\mathrm{X}} \alpha_{\mathrm{Y}} C_{12}\left(\omega_{\mathrm{s}}\right)\right]
\end{aligned}
$$


a.

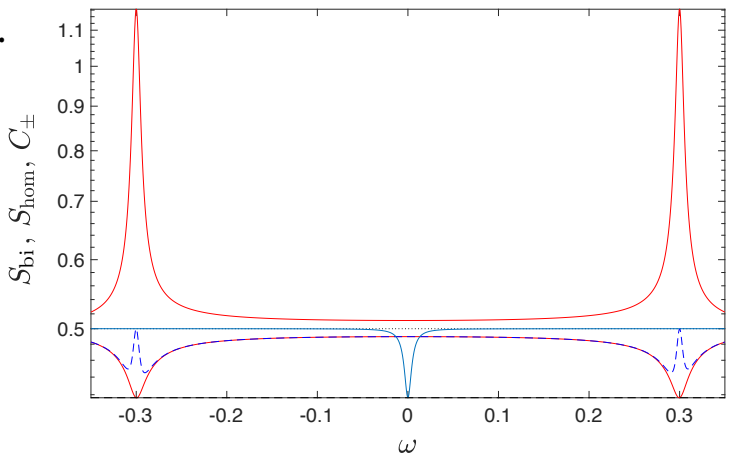

c.

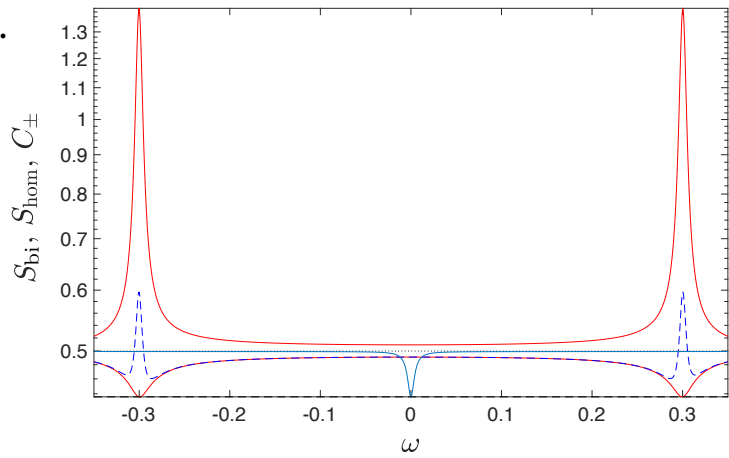

b.

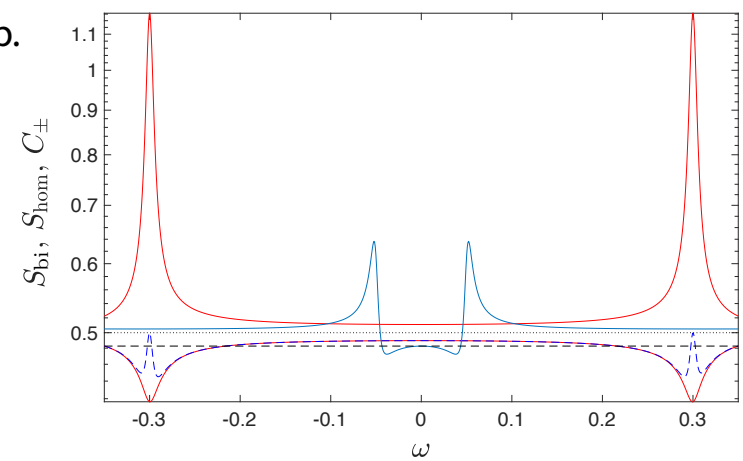

d.

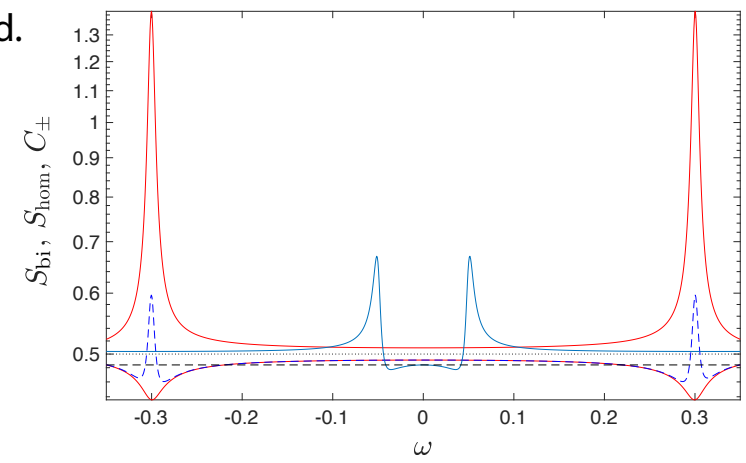

FIG. S1. Comparison between the bichromatic spectrum $S_{\text {bi }}$ (blue line), the homodyne spectrum $S_{\text {hom }}$ (blue dashed line) and the eigenvalues of $C_{\mathrm{i}, \mathrm{j}}\left(C_{ \pm}\right.$, red line $)$for $\omega_{\mathrm{m}} / \kappa=0.3$. a. $\Delta=0, \omega_{\mathrm{s}}=\omega_{\mathrm{m}} ; \mathbf{b} . \Delta=0, \omega_{\mathrm{s}}=1.003 \omega_{\mathrm{m}} ; \mathbf{c} . \Delta / \kappa=-0.2, \omega_{\mathrm{s}}=\omega_{\mathrm{m}}$; d. $\Delta / \kappa=-0.2, \omega_{\mathrm{s}}=1.003 \omega_{\mathrm{m}}$.

The relation given by Eq. (S14) allows us to interpret $S_{\mathrm{bi}}(\omega)$ as a quadratic form associated with the matrix $C_{\mathrm{ij}}(\omega)$ in the variables $\alpha_{\mathrm{X}}$ and $\alpha_{\mathrm{Y}}$. Moreover, setting an arbitrary $\omega_{\mathrm{s}}$, the measurement $S_{\mathrm{bi}}(0)$ allows us to access the smallest (largest) eigenvalue of $C_{\mathrm{ij}}\left(\omega_{\mathrm{S}}\right)$ by choosing $\alpha_{\mathrm{X}}, \alpha_{\mathrm{Y}}$ in such a way that the vector $\left(\alpha_{\mathrm{X}}, \alpha_{\mathrm{Y}}\right)^{\mathrm{T}}$ correspond to the eigenvector associated to the smallest (largest) eigenvalue of $C_{\mathrm{ij}}\left(\omega_{\mathrm{m}}\right)$, thus allowing us to access correlations hidden to homodyne detection. The special case $\omega_{\mathrm{s}}=\omega_{\mathrm{m}}$ essentially accesses the mechanical resonant frequency that shows the strongest noise reduction, and at the same time, the best sensitivity to external forces. Setting the two LO's exactly at the mechanical sidebands at either side of the pump tone is therefore the most relevant case. This case corresponds to the $\mathrm{x}$-axis coordinate equal to zero in Fig. 5 in the main text.

In Fig. S1 we have compared the bichromatic and homodyne spectra $S_{\mathrm{bi}}$ and $S_{\mathrm{hom}}$ with the eigenvalues of $C_{\mathrm{i}, \mathrm{j}}$ for $\Delta=0$ and $\Delta / \kappa=0.3$. We also consider the case $\omega_{\mathrm{s}} \neq \omega_{\mathrm{m}}$. From these plots it is possible to see how the frequency mismatch raises the value $S_{\mathrm{bi}}(0)$, while leaving the eigenvalues of $C_{\mathrm{i}, \mathrm{j}}$ and the homodyne signal unaltered. Conversely, the choice $\Delta \neq 0$ affects the value of the homodyne signal at the mechanical resonance $\omega_{\mathrm{m}}$, increasing its value above the SQL. In the experiment, we chose a slightly red-detuned $\Delta$ in order to broaden the hidden window as compared to exact zero detuning, hence making it more clearly visible.

\section{DATA ANALYSIS}

We use the convention that variables labeled as $S_{\mathrm{o}}$ (with the subscript o) are the spectra right after the sample in units of quanta. The corresponding spectra at the HEMT input are labeled by $N$. These are not the same, because there are some losses, characterized by the power transmission coefficient $t<1$, between the sample and HEMT. In the case of large noise $S_{\mathrm{o}} \gg 1$, these are related by

$$
N=t S_{\mathrm{o}}
$$

After amplification and detection at the analyzer, we write the spectra with the symbol $S$ (without the subscript o), see Eq. (S19) below. The latter quantity is given in $\mathrm{W} / \mathrm{Hz}$.

In all the experiments, as mentioned in the main text, we run the measurement twice: $S(\omega)$ (or $S^{\theta}(\omega)$ in phasesensitive measurements) with the pump tone on, and in a separate measurement, $S_{\text {off }}(\omega)$ (or $\left.S_{\text {off }}^{\theta}(\omega)\right)$ with the pump 
tone off. Reduction of noise when the pump tone is off signifies squeezing. The noise reduction could in principle be due to artifacts in particular owing to amplifiers' nonlinearity. We take careful precautions to exclude these possibilities. First of all, we cancel the pump tones inside the cryostat between the isolators and the first amplifier by around $30 \mathrm{~dB}$, and check the result does not depend on the amount of canceling. Second, we calibrate most of the parameters independently, except the bath temperatures of the mechanics and the cavity, which both depend on the particular pump setting, and tend to grow as the pump power is increased.

We note that the relevant quantity in both squeezing and sideband cooling at high power, instead of the mechanical bath temperature, is the mechanical decoherence rate given as $\gamma n_{m}^{T}$. In these measurements, one therefore cannot distinguish whether the decoherence is due to heating up of the mechanical bath when pump power is cranked up, or due to an enhanced $\gamma$. For convenience, in the analysis, we fix $\gamma$ which results in the same conclusions as keeping $\gamma n_{m}^{T}$ as the free parameter.

\section{Sideband cooling}

We present the sideband cooling data discussed in this section differently from squeezing discussed elsewhere in this work. In accordance with a common practice, sideband cooling spectra shown are referred back to the plane right after the sample $\left(S_{\mathrm{o}}\right)$. The squeezing spectra $(N)$ are those directly detected in front of the HEMT amplifier, which approach involves less inference.

We can apply the single pump tone at considerably varying detunings around the cavity, and thereby obtain information that is inaccessible in the main experiment where the detuning is small, i.e. $|\Delta| \gg \omega_{m}$. The sideband cooling, where we have a red detuning $\Delta \simeq-\omega_{m}$, is the basic such calibration. The sideband cooling in our bad cavity case $\omega_{c}>\omega_{m}$ is not too effective in cooling the mechanics, but we can determine the effective coupling versus the generator power setting, as well as the gain of the measurement system.

When the red-detuned pump power is increased, the mechanics effective linewidth grows, while in the relevant regime the lineshape stays Lorenzian. The linewidth, or "optical" damping, of the mechanics is given by

$$
\gamma_{\mathrm{opt}}=\frac{4 G^{2}}{\kappa}=\frac{4 g_{0}^{2} n_{c}}{\kappa}=\frac{4 g_{0}^{2} \frac{\beta P_{P} \kappa}{\hbar \omega_{c}} \frac{1}{\Delta^{2}+\left(\frac{\kappa}{2}\right)^{2}}}{\kappa}=\underbrace{\frac{4 g_{0}^{2} \beta}{\hbar \omega_{c}}}_{\equiv \mathcal{J}} \frac{P_{P}}{\Delta^{2}+\left(\frac{\kappa}{2}\right)^{2}},
$$

where we used

$$
n_{c}=\frac{\beta P_{P} \kappa}{\hbar \omega_{c}} \frac{1}{\Delta^{2}+\left(\frac{\kappa}{2}\right)^{2}},
$$

and $P_{P}$ is the pump generator power setting, $\beta$ is the attenuation preceding the sample, and $\mathcal{J}$ is the calibration coefficient to be determined. We separate the detuning-dependent cavity response from the fit because several detunings are used. The effective coupling is then

$$
G=\sqrt{\frac{\kappa \gamma_{\mathrm{opt}}}{4}}=\sqrt{\frac{\kappa \mathcal{J} P_{P}}{4\left[\Delta^{2}+\left(\frac{\kappa}{2}\right)^{2}\right]}}
$$

The output spectrum at the sample plane $S_{\mathrm{o}}(\omega)$ for sideband cooling is calculated numerically taking into account bad-cavity effects. The measured pump output spectrum $S(\omega)$ (in W/Hz) on top of the noise floor is

$$
S(\omega)=\underbrace{\mathcal{G} \hbar \omega_{c}}_{\equiv \mathcal{A}} S_{\mathrm{o}}(\omega)
$$

where $\mathcal{G}$ is the gain of the detection system including amplifiers and cable losses, and $\mathcal{A}$ is the calibration coefficient to be determined from the fit. Here, in $S_{\mathrm{o}}(\omega)$ we use the effective coupling calibrated in Eq. (S18).

From a set of spectra measured at varying generator powers (Fig. S2), we first obtain the conversion of generator power into effective coupling (Eq. (S18)), the gain of the detection system, as well as the temperatures of the mechanics and of the cavity under these pumping conditions. All of these can be obtained with very small statistical errors, viz. $\sim 6 \%$ and $10 \%$ for $n_{m}^{T}$ and $n_{c}^{T}$, respectively, in the relevant power region. 
(a)
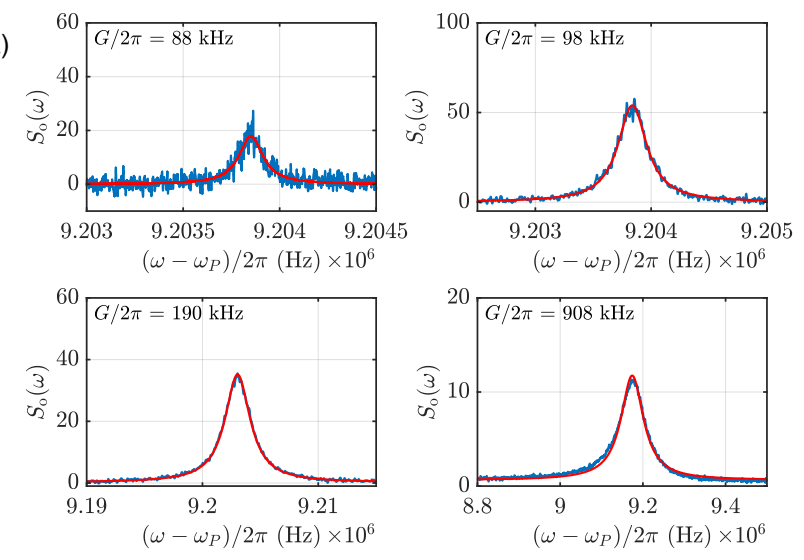

(b)

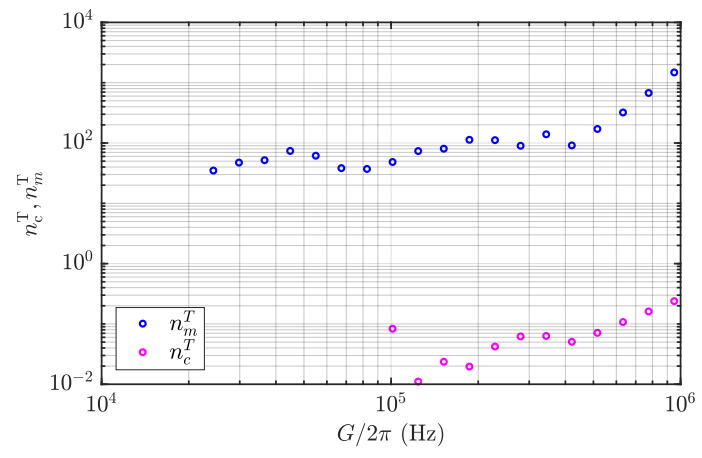

FIG. S2. Sideband cooling. (a), Spectra in units of quanta as calibrated in the circuit plane right after the sample, and theory fits at the effective coupling values quoted in the panels. (b), Extracted bath temperatures of the mechanical oscillator and of the cavity.

\section{Amplifier noise}

The noise floor in the measurement, supposing it is set by the HEMT noise, and by the transmission $t<1$ between the sample and HEMT, is given as

$$
S_{\mathrm{o}}^{\mathrm{eff}}=\frac{N_{\mathrm{HEMT}}}{t}
$$

This is the effective input noise in the measurement, setting e.g. the signal-to-noise ratio. The effect of the transmission here is that it attenuates the signal, although it does not directly affect the noise.

In an earlier cooldown, we have measured $S_{\mathrm{o}}^{\mathrm{eff}} \simeq 18$ at the same frequency [2]. We have not, however, directly measured $t$, whose uncertainty then sets significant error bars for the squeezing. In our system with a standard superconducting coaxial cabling, we estimate a typical $t \approx 1.5 \ldots 3 \mathrm{~dB}$. If for some reason the attenuation would be larger than we estimate, the squeezing would be stronger than claimed now. The attenuation is unlikely to be smaller than the stated values because even if all components are working ideally, the total attenuation amounts to approximately $1.5 \mathrm{~dB}$.

After calibrating the gain with sideband cooling, using Eq. (S19) we can also get an estimate for $S_{\mathrm{o}}^{\text {eff }} \simeq 22$, which is close to the value quoted immediately above.

\section{Squeezing}

The calibration of the squeezing is done by using as a reference the noise level in one quadrature $S_{\text {off }}^{\theta}$ when the pumps are off, corresponding to half the true input noise $N_{\text {HEMT }}=2 N_{\text {HEMT }}^{\theta}$ of the HEMT. When the pumps are on 


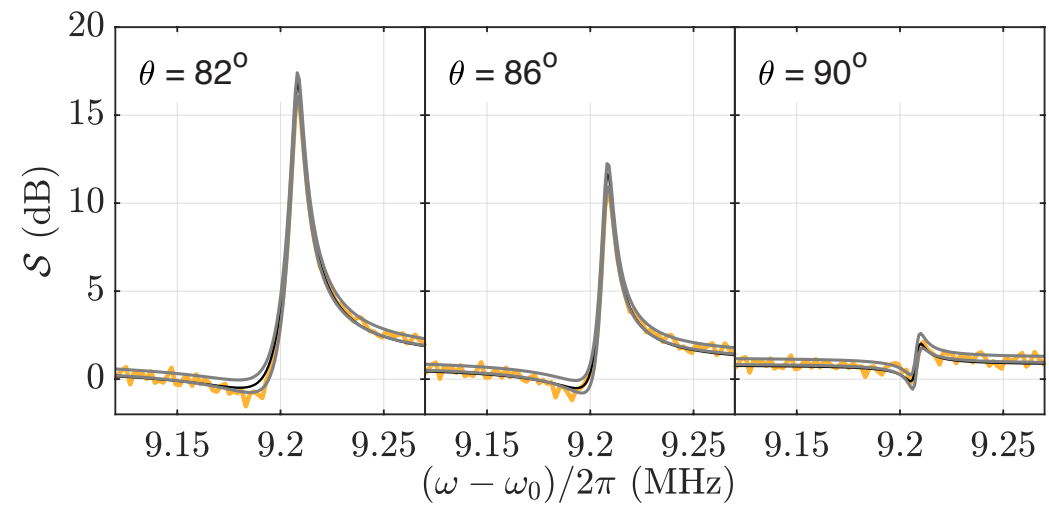

FIG. S3. Squeezing error analysis. Graphs from Fig. 2b, overlaid with the theoretical fit, and error lines (see text).

or off, respectively, the measured noise is

$$
\begin{aligned}
S^{\theta} & =\mathcal{G}\left(N^{\theta}+N_{\mathrm{HEMT}}^{\theta}\right), \\
S_{\text {off }}^{\theta} & =\mathcal{G}\left(N_{z p}^{\theta}+N_{\mathrm{HEMT}}^{\theta}\right) .
\end{aligned}
$$

Here, $N^{\theta}$ is the quantity of interest, the squeezed noise radiating into the HEMT input.

The amount of squeezing is conveniently expressed as the noise in one quadrature in units of $N_{z p}^{\theta}=\frac{1}{4}$ :

$$
\mathcal{S}=\frac{N^{\theta}}{N_{z p}^{\theta}}
$$

and hence a value $<1$ (or $0 \mathrm{~dB}$ ) entails squeezing below vacuum. Using Eqs. (S21,S22), Eq. (S23) becomes

$$
\mathcal{S}=\frac{S^{\theta} / \mathcal{G}-N_{\mathrm{HEMT}}^{\theta}}{N_{z p}^{\theta}}=\frac{1}{N_{z p}^{\theta}}\left[\frac{S^{\theta}}{S_{\mathrm{off}}^{\theta}}\left(N_{z p}^{\theta}+N_{\mathrm{HEMT}}^{\theta}\right)-N_{\mathrm{HEMT}}^{\theta}\right]
$$

From the theory, we obtain the squeezing directly at the output of the sample $\left(S_{\mathrm{o}}^{\theta}\right)$. Losses, however, reduce the amount of squeezing by bringing the state towards a thermal state. In order to compare the data to theory, we therefore have to propagate the theory to the observation plane. The general version of Eq. (S15) that holds for arbitrary $N$ is then

$$
N^{\theta}=t S_{\mathrm{o}}^{\theta}+N_{z p}^{\theta}(1-t)
$$

which we use to obtain the theoretical predictions for squeezing.

The error bars of the ponderomotive squeezing as measured by the HEMT are estimated from two contributions. One is a statistical uncertainty in the relevant frequency range in the curves in Fig. 2 of the main text. For example, in Fig. 2 (b) middle panel, the range is about $9.185 \ldots 9.195 \mathrm{MHz}$. The other source of error is the uncertainty in the transmission $t$, which has the effect of stretching the vertical axis of the squeezing spectra about the zero decibel level. The error in $t$ is hard to quantify, but as a worst-case estimate we select $\pm 1.5 \mathrm{~dB}$. Notice that uncertainty in $t$ alone cannot raise the squeezing level above vacuum. These two error contributions are propagated in Eq. (S24).

At approximately zero detuning, we cannot measure the effective coupling independently, but we use Eq. (S18) under the considered $\Delta$ to get a prediction for the $G$.

Regarding the bath temperatures, the final values obtained for the mechanics are close to those from sideband cooling. For the cavity, the value in the latter is somewhat lower at the same $G$. In our samples, the bath temperatures can depend sensitively on the pumping conditions (frequency and power), as seen in Fig. S2, so differences can be anticipated.

It would be interesting to evaluate error bars for the squeezing based on the parameters obtained by independent means. Here we use the bath temperatures from sideband cooling (Fig. S2) at the same $G$, and combine their errors doubled either worst-case or best-case. The result, as shown in Fig. S3, demonstrates that squeezing is rather robust against changes in bath temperatures. 
We now briefly discuss how the data points in Fig. 5 in the main text are determined. They are the minima in the spectra in Fig. 4, plus more curves not shown in Fig. 4. Ideally, each point in Fig. 5 would be the value of a spectrum in Fig. 4 at the $\mathrm{x}$-axis value $=0$. In the real case because of noise, of course, we have to average several points in the spectrum to get one point to Fig. 5. The averaging range around zero is to some extent arbitrary. A large range would drop the error bars because of more information, but would compromise the obtained squeezing, because the squeezing deteriorates away from zero. We chose the averaging range such that a corresponding theory curve would predict less than around $20 \%$ reduction of squeezing as compared to zero frequency. At small values of $\omega_{s}-\omega_{m}$, this corresponds to only a few datapoints in the curves as in Fig. 4. The error bars, however, are obtained from non-smoothed data, so that more than around 10 independent data points are considered in each case in Fig. 5. The $\pm 2 \sigma$ error bars then become approximately twice the standard error of those points.

* mika.sillanpaa@aalto.fi

[1] L F Buchmann, S Schreppler, J Kohler, N Spethmann, and D M Stamper-Kurn, "Complex Squeezing and Force Measurement Beyond the Standard Quantum Limit," Phys. Rev. Lett. 117, 030801-9 (2016).

[2] C. F. Ockeloen-Korppi, E. Damskägg, J.-M. Pirkkalainen, T. T. Heikkilä, F. Massel, and M. A. Sillanpää, "Noiseless quantum measurement and squeezing of microwave fields utilizing mechanical vibrations," Phys. Rev. Lett. 118, 103601 (2017). 